\title{
Exploring peptide capture by anti-protein antibodies for LC- MS-based biomarker determination
}

\author{
Maren C. S. Levernæs ${ }^{1}$, Bassem Farhat ${ }^{1}$, Inger Oulie ${ }^{1}$, Sazan S. Abdullah ${ }^{1}$, Elisabeth Paus ${ }^{2}$, Léon \\ Reubsaet $^{1}$, Trine G. Halvorsen ${ }^{1}$ * \\ ${ }^{1}$ Department of Pharmaceutical Chemistry, School of Pharmacy, University of Oslo, Norway \\ ${ }^{2}$ Department of Medical Biochemistry, Norwegian Radium Hospital, Oslo University Hospital, Norway
}

\section{Abstract}

Immunocapture LC-MS/MS is a promising technique to ensure high sensitivity and selectivity of lowabundant protein biomarkers. For this purpose, the use of monoclonal antibodies (mAb) is especially attractive as they are renewable reagents that can be standardized. In this article we investigated the possibility of using mAbs developed against intact proteins (anti-protein antibodies) to capture proteotypic epitope peptides. Three mAbs were tested, and all selectively extracted proteotypic epitope peptides from a complex sample. Compared to intact protein extraction, this concept which we call peptide capture by anti-protein antibodies provided cleaner extracts, which further improved the sensitivity. Analysis of three patient samples demonstrated that $p$ can be used for the determination of different endogenous protein levels.

\section{Introduction}

In recent years the proteomics society has shown a growing interest in targeted mass spectrometry (MS) based methods to quantify protein biomarkers ${ }^{1}$. Biomarkers have big potential in the medical field by providing diagnostic or prognostic tools, assessing risk profiles and outcomes by defining disease states, and contributing to personalized therapeutic strategies. Most clinical applications are focused on blood biomarkers, and due to the low dynamic range of proteomic technology, samples must be depleted for highly abundant proteins in order to detect low-abundant protein biomarkers ${ }^{2}$. Despite its unique mass selectivity, sensitive detection of intact proteins by MS is limited. The protein biomarker is thus usually digested by a proteolytic enzyme to generate proteotypic peptides that are analyzed by LC-MS ${ }^{3-4}$. The choice of the surrogate peptide, also known as the signature peptide or proteotypic peptide, used for the quantification of the entire protein is crucial for the quality of the assay and should thus be unique to the targeted $\operatorname{protein}^{3-5}$. 
Among the most promising methods to ensure high sensitivity and selectivity in targeted analysis of low-abundant protein biomarkers is a combination of immunoaffinity enrichment (immunocapture), protein digestion and LC-MS/MS detection of signature peptides ${ }^{1,3-7}$. Immunocapture provides unique selectivity through the unique interaction of the target protein or signature peptide and the capture antibody ${ }^{3}$. These antibodies target either linear or conformational epitopes. Linear epitopes consist of continuously neighbouring amino acid residues along the protein sequence, while conformational epitopes consist of amino acid residues that are discontinuously arranged along the protein sequence, that are brought together through folding of the polypeptide chain ${ }^{8}$. Thus antibodies targeting conformational epitopes are used for the enrichment of the intact protein, whereas antibodies targeting linear epitopes may both capture intact protein and epitope containing peptides $^{6}$. Additionally, anti-peptide antibodies may be synthesized to target signature peptides $(\text { SISCAPA })^{4,6}$.

The greatest advantage of choosing peptide immunoenrichment over protein immunoenrichment is that peptide samples are easier to handle, due to degradation, unfolding and solubility issues often associated with full-length proteins ${ }^{6}$. Quantitative, multiplexed immuno-MRM assays targeting peptides rather than proteins are expected to produce cleaner eluates and thus less interfering peptides are introduced to the LC-MS ${ }^{9}$. In addition, as the proteolytic step is performed prior to extraction, the antibodies may be reused and thus the cost per sample is reduced. Approaches based on peptide capture are also less likely to be influenced by autoantibodies, as these antibodies are destroyed during the proteolytic step ${ }^{10}$.

The use of monoclonal antibodies (mAbs) is especially attractive for both peptide and protein enrichment as they are renewable reagents that can be standardized, in contrast to polyclonal antibodies that are a limited source suffering from batch-to-batch variations ${ }^{9}$. The availability of validated mAbs for immunoaffinity enrichment of proteotypic peptides is however limited ${ }^{11}$. Antiprotein antibodies, on the other hand, are often commercially available due to ongoing antibody screening in pharmaceutical industry ${ }^{1}$. Hence, peptide capture by anti-protein antibodies might be a promising alternative for the enrichment of proteotypic peptides without the need for timeconsuming and expensive development of anti-peptide antibodies. In 2004, Zhao et al. characterized the epitope of an anti-troponin antibody by epitope excision and the identified (missed cleavage) peptide was used as the surrogate peptide in the targeted protein quantification ${ }^{12}$. This peptide capture by anti-protein antibodies (PeCAPrA) approach is most easily applied to antibodies with known epitopes. However, as recently demonstrated by Schoenherr et al. it can also be applied without knowledge of the epitope configuration (overall success rate $14 \%)^{9}$. In order to successfully develop a peptide capture assay based on anti-protein antibodies, the antibody should recognize a 
linear epitope, not disrupted by a cleavage site. Since the majority of the commercially available antibodies used for Western blot analysis and ELISA assays have linear epitopes ${ }^{13}$, this approach is predicted to be widely applicable.

In the presented study, peptide capture by anti-protein antibodies was demonstrated using wellcharacterized mAbs with known epitopes. Antibodies used for the immunocapture of an intact protein biomarker (Pro-gastrin releasing peptide, ProGRP) were used as model system due to the availability of several mAbs. In addition, a method based on immunocapture of the intact protein followed by LC-MS/MS determination of signature peptides ${ }^{14-15}$ was available as a reference for the evaluation of the new approach.

Peptide capture by anti-protein antibodies was investigated using three different mAbs (E146, E149, and M18) developed against intact ProGRP. The following parameters were evaluated: The possibility to produce epitope containing peptides using commonly applied digestion enzymes, challenges related to performing the digestion prior to the immunocapture step, linearity, and limits of detection (LOD) and quantification (LOQ). In addition, a brief comparison with intact protein extraction was performed with emphasis on the cleanness of the extracts, extraction yield, and LODs and LOQs. Finally, to assess the feasibility of using the epitope peptide as the surrogate peptide in the quantification of proteins in real samples the low abundance model biomarker ProGRP was determined in serum; both in spiked samples and samples from patients diagnosed with small cell lung cancer (SCLC).

\section{Results and discussion}

\section{Selection of signature epitope peptides}

A signature peptide used for the quantification of a protein should uniquely identify the targeted protein, be about 7-23 amino acids long, and suitable for QqQ analysis in MRM mode ${ }^{5,16}$. Peptides containing or susceptible to post-translational or chemically introduced modifications should be avoided, in addition to missed cleavage peptides ${ }^{16}$. When targeting proteotypic peptides containing the epitope of mAbs produced against intact proteins however, the choice of surrogate peptides is limited to peptides containing the intact epitope recognized by the antibody. The antibodies used would have to target linear epitopes and the epitope should be protein specific. As a result the peptide of interest might not be the proteotypic peptide with highest sensitivity during screening. However, due to the efficiency of the selected mAb and the excellent clean-up using PeCAPrA (as described below) the desired detection limits might still be possible to reach. 
As mentioned earlier, peptide capture by anti-protein antibodies is most easily applied to antibodies with known epitopes. For the model system applied in the present paper Nordlund et $\mathrm{al}^{17}$ have successfully produced several mAbs with very low equilibrium dissociation constants (between $2.63 \times 10^{-10}$ and $1.00 \times 10^{-9}$, reflecting very high affinity) which recognise and bind to different regions of our model protein ProGRP (figure 1). Amongst these, mAb E146 had the lowest equilibrium dissociation constant $\left(2.63 \times 10^{-10}\right)$ and was shown to be the best capture antibody. mAb E146 recognizes a linear epitope covering amino acid residues 48-53 (LREYIR) and in combination with mAb E149, which recognize a different epitope covering amino acid residues 84-88 (QQPSW), a very sensitive two-sided immunofluorometic assay (IFMA) has been developed ${ }^{18}$. The affinity of mAb E149 seems to be affected by conformational changes of ProGRP and other mAbs recognizing the same epitope has also been developed ${ }^{17}$. Amongst these, mAb M18 was proven to be a good tracer outperforming the original tracer antibody, $\mathrm{E} 149^{19}$.To investigate if an epitope containing zero missed cleavage peptide could be generated for mAb E146 and mAbs E149/M18, in silico digests with different enzymes (trypsin, chymotrypsin and Lys-C) were performed in Protein Prospector. This search resulted in one signature epitope peptide candidate for E146 using Lys-C and one for E149 and M18 using trypsin and Lys-C. The following BLAST query showed that the only source for both peptides was ProGRP. Finally SEQUEST identified the signature epitope peptide candidates performed on data from FT-Orbitrap analysis on in solution digests of ProGRP. Thus the Lys-C generated peptide QQLREYIRWEEAARNLLGLIEAK and the trypsin (and Lys-C) generated peptide ALGNQQPSWDSEDSSNFK were chosen as signature epitope peptides for mAb E146 and mAbs E149/M18, respectively.

\begin{tabular}{|c|c|c|c|c|c|c|c|c|c|c|c|c|c|c|c|c|c|c|c|c|c|c|c|c|c|c|c|c|}
\hline V & $\mathrm{P}$ & $\mathrm{L}$ & $P$ & A & G & G & $\mathrm{T}$ & V & $\mathrm{L}$ & $\mathrm{T}$ & K & $\mathrm{M}$ & $Y$ & $\mathrm{P}$ & R & G & $\mathrm{N}$ & $\mathrm{H}$ & W & A & V & G & $\mathrm{H}$ & $\mathrm{L}$ & $\mathrm{M}$ & G & K & K \\
\hline & & & & & & & & & & & & & & & & & & & & & & & & & & & & $\frac{S}{T}$ \\
\hline L & $\mathrm{L}$ & $\mathrm{N}$ & $R$ & A & A & $E$ & $E$ & W & $\mathrm{R}$ & 1 & $\mathrm{Y}$ & $E$ & $\mathrm{R}$ & $\mathrm{L}$ & $\mathrm{Q}$ & Q & $\mathrm{K}$ & L & $\mathrm{S}$ & G & $\mathrm{R}$ & $E$ & $\mathrm{~S}$ & V & $\mathrm{S}$ & $\mathrm{S}$ & $E$ & $\mathrm{G}$ \\
\hline $\mathrm{G}$ & & & & & & & & & & & & & & & & & & & & & & & & & & & & \\
\hline $\mathrm{L}$ & & & & & & & & & & & & & & & & & & & & & & & & & & & & \\
\hline 1 & $E$ & A & $\mathrm{K}$ & $E$ & $\mathrm{~N}$ & $R$ & $\mathrm{~N}$ & $\mathrm{H}$ & $\mathrm{Q}$ & $P$ & $P$ & $\mathrm{Q}$ & $P$ & $\mathrm{~K}$ & A & $\mathrm{L}$ & G & $\mathrm{N}$ & $\mathrm{Q}$ & $\mathrm{Q}$ & $P$ & $\mathrm{~S}$ & W & D & $\mathrm{S}$ & $E$ & D & $\mathrm{S}$ \\
\hline & & & & & & & & & & & & & & & & & & & & & & & & & & & & $\frac{S}{N}$ \\
\hline $\mathrm{Q}$ & $\mathrm{N}$ & L & $\mathrm{Q}$ & $P$ & $N$ & $\mathrm{R}$ & G & $E$ & $\mathrm{R}$ & $\mathrm{Q}$ & $S$ & G & $P$ & A & $S$ & $\mathrm{~L}$ & $R$ & G & V & K & G & K & $S$ & G & V & D & K & $F$ \\
\hline
\end{tabular}

Figure 1. ProGRP isoform 1 sequence with binding epitopes of available anti-ProGRP mAbs The binding epitopes have been determined by pepscan analysis. Linear epitopes: - E172, - E146, - M11 and M16, - E149, M7, M8, M9, M15, M18 and M19, and - M37. Non-linear epitopes: - E168. Trypsin cleavages sites are marked with red (lysine) and green (arginine) letters. 


\section{Evaluation of mAbs for epitope peptide extraction}

To demonstrate that the antibodies were capable of extracting epitope containing peptides, immunocapture of an in solution digest of ProGRP was performed, using Lys-C or trypsin for E146 and E149/M18 respectively. All three anti-protein mAbs could be used for epitope peptide extraction as they selectively extracted peptides containing the epitope from a digested sample (Figure S1). However, the epitope peptide of E146 is rather long (23aa; QQLREYIRWEEAARNLLGLIEAK) and a high concentration $(2.5 \mu \mathrm{g} / \mathrm{mL})$ was needed to obtain a good $\mathrm{MS}$ signal. This, in addition to an extraction yield of $31 \%$, would make it difficult make a sensitive assay based on PeCAPrA with mAb E146. The epitope peptide of E149/M18 on the other hand, produced a good MS signal at a lower concentration $(250 \mathrm{ng} / \mathrm{mL}$ ) compared to the epitope peptide of E146. The extraction yield of E149 was however considerably lower than the extraction yield of M18, as also shown by Nordlund et $\mathrm{al}^{19}$, with respectively $15 \%$ and $95 \%$. The observed difference may be due to conformational changes upon digestion that may affect the antibody's affinity towards the epitope. This demonstrates the importance of conformation of the target molecule for efficient extraction. In addition to the zero missed cleavage peptide, two missed cleavage peptides were extracted with M18 and E149. Incomplete digestion and differing production of a peptide can be a challenge when used as a signature peptide for quantification of the intact protein. The production of the zero missed cleavage peptide and the missed cleavage peptides containing the epitope of M18/E149 was however reproducible $(4.7 \% R S D, n=5)$. These results shows great promise for the successful development of a PeCAPrA assay; peptide immunoenrichment assay based on monoclonal antibodies produced for the immunocapture of intact proteins, with ProGRP and mAb M18 as model system.

\section{Method refinement and verification}

\section{Effect of protease on antibody performance}

One challenge related to immunocapture of proteolytic peptides is the presence of active enzyme in the sample during extraction that may compromise the performance of the antibody. To investigate if the presence of active enzyme had any impact on the extraction yield the antibody coated magnetic beads E146 and M18/E149 were incubated with active Lys-C $(6.25$ and $250 \mathrm{ng} / \mathrm{mL})$ or trypsin $(6.25 \mathrm{ng} / \mathrm{mL}, 250 \mathrm{ng} / \mathrm{mL}$ and $50 \mathrm{mg} / \mathrm{mL})$, respectively, for 2 hours at room temperature prior to extraction of intact protein. After incubation, the antibody containing beads were washed and used to extract intact ProGRP from spiked samples $(250 \mathrm{ng} / \mathrm{mL})$, followed by on-beads trypsin digestion overnight. The quantitative yield of the signature peptides generated after digestion were compared to those generated from an extraction of intact ProGRP by antibody containing beads not exposed to active enzyme prior to extraction (control). 
There was, as shown in figure 2, a significant decrease in the quantitative yield of ProGRP after the extraction by antibodies exposed to active enzyme compared to the control ( $P$-value $<0.05)$, except for the E146 beads which was more resistant towards low amounts of active enzyme and showed no significant difference in the quantitative yield compared to the control ( $P$-value $>0.05)$. When exposed to the amount of enzyme needed to digest a protein precipitated serum sample (50 $\mathrm{mg} / \mathrm{mL}$ ), the antibodies still worked, but their performance (monitored by signal intensity of signature) was reduced to about $20-30 \%$. The observed decrease is most likely due to digestion of the antibody. When targeting low abundant protein biomarkers, high extraction yield is needed in order to reach sufficient detection and quantification limits. Thus alternative digestion procedures would be beneficial to preserve the antibodies performance.

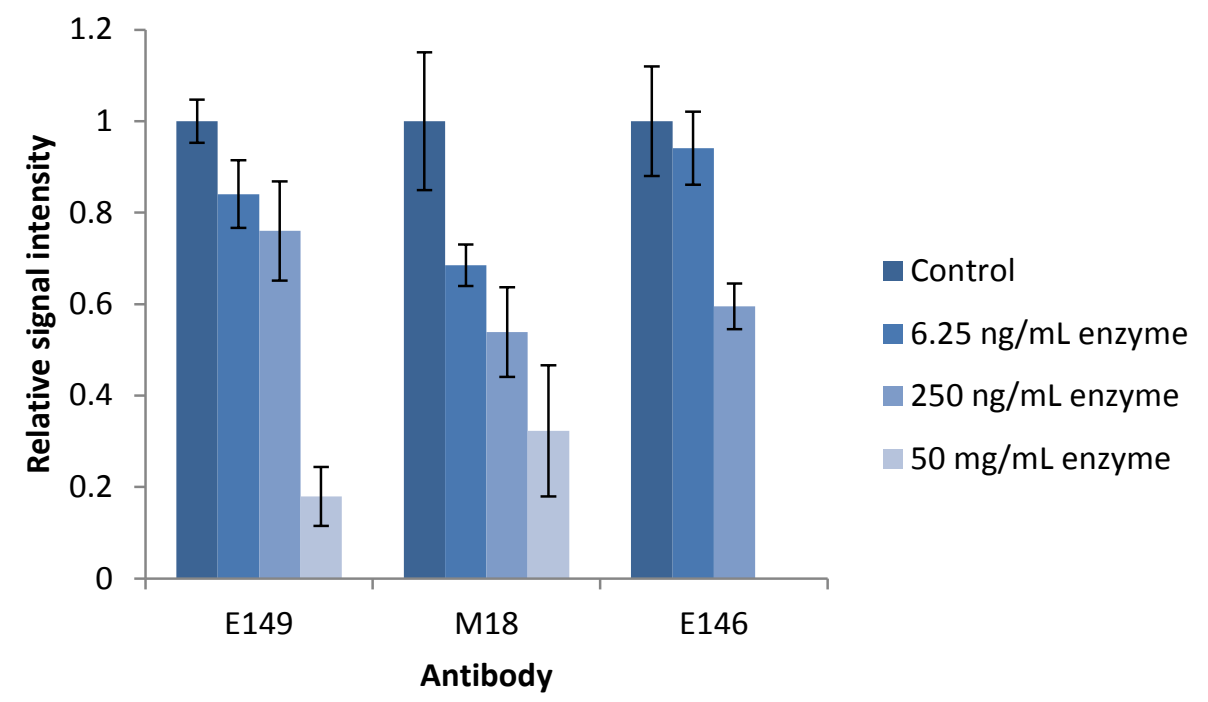

Figure 2. Effect of protease on antibody performance. The antibodies were incubated with active enzyme in an enzyme-to-antibody ratio of 1:1280 (6.25 ng), 1:32 (250 ng) and 6250:1 (50 mg) for 2 hours prior to extraction of intact ProGRP $(250 \mathrm{ng} / \mathrm{mL}, \mathrm{n}=3)$. mAb E149 and M18 were incubated with trypsin while mAb E146 was incubated with Lys-C. Antibody performance was evaluated by immunocapture of intact ProGRP followed by digestion on-beads overnight and subsequent LC$\mathrm{MS} / \mathrm{MS}$ analysis. Error bars represents the standard deviation. *Significantly different from the control (P-value $<0.05)$.

This challenge is also true for SISCAPA assays and different SISCAPA assays have solved this by adding TLCK (tosyl-L-lysine chloromethyl ketone) ${ }^{20-21}$ to stop the trypsin activity or by adding acid and performing an additional clean-up step (SPE, spin-filter, evaporation) $)^{22-25}$ prior to extraction. In the research presented here we circumvent the issue by using trypsin immobilized on beads, which allowed us to remove trypsin from the sample prior to extraction. Immobilization of active enzyme on beads provides improved digestion efficiency due to the increased enzyme-to-protein ratio, 
enabling shorter digestion time. In addition, the immobilization leads to increased enzyme stability through reduced autolysis, thus the beads can be regenerated and reused several times without compromising the proteolytic activity ${ }^{26}$.

\section{Extraction from complex samples}

Even though the selective extraction of epitope containing peptides from an in solution digest in buffer was very promising, extraction from more complex samples was needed to confirm the results. Initially, the possibility of digesting ProGRP spiked serum samples directly using trypsin beads, without any kind of depletion, was briefly investigated using mAb M18. This resulted in a clear but relatively low signal for the signature peptide ALGNQQPSWDSEDSSNFK ( $33 \%$ of the signal obtained after optimization of the method, data not shown). These results suggested that it would be possible to perform the extraction of digested ProGRP from complex samples after optimization of the digestion procedure. As protein precipitation has successfully been used in the sample cleanup of ProGRP previously ${ }^{27-28}$, it was included in the current work as well. ProGRP is a small protein that, with the use of the right amount of acetonitrile, will remain in the acetonitrile fraction after precipitation. Thus the final sample preparation involved protein precipitation, digestion (with enzyme immobilized on beads), epitope peptide extraction and elution. When targeting bigger proteins, this approach may however not be feasible and digestion protocols like the recently described 'addition only' by Razavi et al ${ }^{20}$ should be considered.

Following the initial peptide extraction from a complex sample, three different experiments was performed, primarily to confirm that selective peptide capture by anti-protein antibodies from complex samples was possible but also to make a survey of potential loss of analyte. First, extraction of Lys-C or trypsin digested ProGRP added to protein precipitated and trypsin digested serum was performed. This would demonstrate that epitope peptide extraction from complex samples after protein precipitation was possible. Second, extraction from a sample where intact ProGRP was added to protein precipitated serum prior to tryptic digestion was performed. This would prove that ProGRP could be digested (with trypsin) and extracted from a more complex sample. Finally, representing the final and complete sample preparation, intact ProGRP was added to serum prior to protein precipitation, followed by tryptic digestion and epitope peptide extraction. For mAb E146, only the first experiment was performed as it was too expensive to make beads immobilized with Lys-C.

The results showed that it was possible to extract the epitope peptides from a complex sample using all three mAbs. A decrease in the signal intensity of the epitope peptide was however observed after extraction with both E149 and M18 with increased sample preparation complexity. This indicates a 
loss of analyte both during protein precipitation and digestion. For M18, the major contributor to the loss of signal intensity was the digestion, with a recovery of $52 \%$ (14\% RSD), while protein precipitation was the major contributor to the between sample variation, with $25 \%$ RSD (54\% recovery). The same trend was observed for E149. The loss of signal intensity due to incomplete digestion was higher than for M18, with a recovery of $38 \%$ (16\% RSD). In addition, the protein precipitation contributed to both an additional loss of signal intensity (18\% recovery) and increased between sample variations, with $28 \%$ RSD. These results indicated that both the digestion and protein precipitation step could be optimized in order to increase the digestion efficiency and reduce between sample variations.

\section{Method optimization}

In order to detect the low levels of endogenous ProGRP in serum, optimization of both digestion (with trypsin beads) and protein precipitation were performed. This optimization was done only with mAb M18 as it outperformed mAb E149 based on extraction yield and thus signal intensity of the epitope peptide.

\section{Digestion}

Initial experiments with immobilized trypsin were performed using $10 \mu \mathrm{L}$ beads. In order to ensure efficient digestion, optimization was performed by comparing different amount of beads $(10 \mu \mathrm{L}, 15$ $\mu \mathrm{L}$ and $30 \mu \mathrm{L}$ ) and different incubation times ( 2 hours, 4 hours, and overnight). The highest signal intensity of the epitope peptide was observed after digestion with $30 \mu \mathrm{L}$ beads. There was however no significant difference between the three time points and to speed up the sample preparation a 2 hour digestion was thus chosen (data not shown).

\section{Protein precipitation}

In order to reduce the between sample variation originating from the protein precipitation step the following was investigated; 1 ) digestion directly in the diluted acetonitrile fraction without the evaporation step and 2) optimization of the amount of acetonitrile used for precipitation. A detailed description of this optimization can be found in the supplementary information. In brief, dilution of the acetonitrile fraction with $A B C$ buffer $(50 \mathrm{mM})$ in a ratio of 1:40 and precipitation with a ratio of 1:0.7 provided the highest signal intensity and the lowest RSD values. These common efforts reduced the between sample variation from $30-50 \%$ RSD to $10 \%$ RSD.

\section{Method evaluation}

A brief evaluation of the method was performed using a triple quadrupole (TSQ Quantiva) demonstrating the linearity, limit of detection (LOD) and limit of quantification (LOQ). To investigate 
if the PeCAPrA method was feasible for detecting varying protein concentrations in serum, extractions from serum with ProGRP concentrations ranging from $500 \mathrm{pg} / \mathrm{mL}$ to $250 \mathrm{ng} / \mathrm{mL}$ was performed ( 7 concentration levels, $n=3$ ) after digestion using mAb M18.

A linear curve $(1 / X)$ with acceptable correlation value $\left(R^{2}=0.995\right)$ was produced for the epitope peptide (ALGNQQPSWDSEDSSNFK) of mAb M18. The \% RSD values were $17.5 \%$ or lower for all seven concentration levels, without the use of an internal standard. For implementation of quantitative methods based on the PeCAPrA concept in a clinical setting implementation of internal standard is necessary. This is expected to further improve the reproducibility especially since the implementation of stable isotopically labeled peptides also will correct for the immunocapture step. However, heavy labeled peptides are rather expensive, and were not available in this study.

From the signal-to-noise ratio of the lowest concentration of the curve, the LOD was estimated to be $54 \mathrm{pg} / \mathrm{mL}(\mathrm{S} / \mathrm{N}=3)$, and the LOQ was estimated to be $181 \mathrm{pg} / \mathrm{mL}(\mathrm{S} / \mathrm{N}=10)$. These results show great promise for a reliable detection and quantification of the protein biomarker based on PeCAPrA.

\section{Comparison with protein extraction}

To further evaluate PeCAPrA as a new and improved concept for protein quantification, a brief comparison of protein and peptide extraction with M18 was performed with emphasis on extraction efficiency, general clean-up efficiency, detection and quantification limits.

\section{Extraction efficiency}

When applying antibodies produced towards intact protein to extract the epitope containing peptide it is of interest to investigate if the antibody is as efficient to extract the epitope peptide as the intact protein. This was evaluated by performing an extraction of intact ProGRP $(100 \mathrm{ng} / \mathrm{mL})$ from buffer $(\mathrm{n}$ $=5$ ). After extraction the unbound fraction was removed from the beads and then both the bound (extracted) and unbound (not extracted) fraction was digested overnight and analyzed by LC-MS/MS. Similarly an extraction of the epitope peptide from an in solution digest of ProGRP (100 ng/mL) was performed $(n=5)$. Again both the bound and unbound fraction was analyzed by LC-MS/MS. The signal intensity of the epitope peptide in the bound and unbound fraction was compared to determine the extraction efficiency. The extraction yield of the intact protein was determined to 99 $\pm 15 \%$, while the extraction yield of the epitope peptide was determined to $996 \pm 6 \%$. These results indicate that there is no significant difference (P-value $>0.05)$ in the extraction efficiency between protein and peptide extraction with mAb M18. This demonstrates that by careful selection of antibody it is possible to achieve similar extraction efficiency for the epitope containing peptide as for the intact protein. 


\section{General clean-up efficiency}

As the extraction efficiency of the epitope peptide was proven to be similar to that of intact protein extraction, PeCAPrA was expected to be more beneficial as it is most likely would produce cleaner extracts. To compare the general clean-up efficiency of the two methods, an extraction of both the intact protein and the epitope peptide from ProGRP $(150 \mathrm{ng} / \mathrm{mL})$ spiked serum was performed and analyzed on a LTQ-Orbitrap ( $n=4)$.

The observed background was, as expected considerably higher after protein extraction and onbeads digestion compared to the extraction of the epitope peptide (Figure S2). One might expect this to be due to the digestion of the antibodies; however, only 11 out of the 111 unique peptides identified originated from the antibody M18. As also shown for on-beads digestion previously ${ }^{29}$, the major contribution to the increased background was the detection of 100 unique peptides from 19 human proteins. In comparison only three unique peptides from serum albumin was identified in addition to the epitope peptide after peptide extraction. These conclusions are also valid when comparing to protein extraction followed by elution of intact protein prior to digestion: ${ }^{29}$ Using mAb E146 for intact protein extraction 115 unique peptides from 32 human proteins were detected after on-beads digestion while elution followed by subsequent digestion resulted in identification of 29 unique peptides from five human proteins. The observed difference may be due to the assumption that smaller proteolytic peptides to a lesser degree bind non-specific to the antibody and/or the magnetic beads compared to intact proteins. Nevertheless, these results demonstrate that peptide extraction provides the highest degree of sample clean-up. Cleaner extracts is expected to provide less matrix effects, which further will improve the sensitivity. Peptide capture by anti-protein antibodies may thus be an advantage in multiplexed assays where several biomarkers are determined at once.

\section{LOD and LOQ}

To compare the detection and quantification limits of PeCAPrA and immunocapture of intact protein an extraction of ProGRP $(5 \mathrm{ng} / \mathrm{mL}$ ) from serum was performed $(n=5)$ and the samples were analyzed on a triple quadrupole (TSQ Qantiva). The signal intensity of the epitope peptide was considerably lower after peptide extraction compared to intact protein extraction. The decrease in signal intensity is probably due to the high sample complexity and thus decreased digestion efficiency, compared to the digestion of an extracted protein, still the estimated LOD $(S / N=3)$ and LOQ $(S / N=10)$ was lower for PeCAPrA (54 pg/mL and $181 \mathrm{pg} / \mathrm{mL}$ respectively as described previously). In comparison, the estimated LOD and LOQ for intact protein extraction were $459 \mathrm{pg} / \mathrm{mL}$ and $1532 \mathrm{pg} / \mathrm{mL}$ respectively. Based on these results PeCAPrA provides a detection limit below the upper reference limit of ProGRP 
in healthy individuals $(58.9 \mathrm{pg} / \mathrm{mL})^{19}$, whereas protein extraction and detection of the same peptide (ALGNQQPSWDSEDSSNFK) with the same antibody (M18) does not.

When comparing with the signature peptide used in immunocapture of intact protein (NLLGLIEAK) ${ }^{14}$ however, PeCAPrA using M18 (and ALGNQQPSWDSEDSSNFK) matches the estimated LOD and LOQ of $48 \mathrm{pg} / \mathrm{mL}$ and $162 \mathrm{pg} / \mathrm{mL}$, respectively.

\section{Analysis of patient samples}

Even though the purpose of this study was to demonstrate the applicability of a new concept for immunocapture of prototypic peptides and not to develop a new assay for the determination of ProGRP, it was important to demonstrate the applicability of PeCAPrA in real samples. Serum samples from both healthy individuals and three patients diagnosed with SCLC $(0.5 \mathrm{ng} / \mathrm{mL}, 1.1 \mathrm{ng} / \mathrm{mL}$ and $2.6 \mathrm{ng} / \mathrm{mL}$ ) were thus analyzed. The epitope peptide was detected in serum from both healthy individuals (figure S3B) and patients suffering from SCLC (figure S3C), demonstrating that the PeCAPrA method can be used for detection of a wide range of ProGRP levels. Compared to the routinely used immunoassays (TR-IFMA), the ProGRP levels estimated using the PeCAPrA LC-MS/MS method was between 1-20 times higher. There may be several reasons for this difference; however, due to the small number of samples no real method comparison can be performed. Nevertheless, these results demonstrated that extraction of the epitope containing peptide ALGNQQPSWDSEDSSNFK was successful also in real samples from patients suffering from SCLC, using anti-protein antibodies.

\section{Conclusions}

In this study we have demonstrated that peptide capture by anti-protein antibodies is a promising method for targeted biomarker determination. This is to our knowledge the first report of an immunocapture LC-MS/MS based assay where antibodies produced against intact proteins has successfully been used for the selective enrichment of epitope containing peptides.

The concept was evaluated using a protein biomarker (ProGPR) as model analyte. The monoclonal antibody M18, developed against intact ProGRP, selectively extracted the epitope peptide from a complex sample. The optimized PeCAPrA method was compared to the previously validated LCMS/MS method for the immunocapture of intact ProGRP and the results demonstrated that peptide extraction provided cleaner extracts, similar sensitivity and hence most likely less matrix effects compared to the extraction of intact protein. These qualities will make PeCAPrA an attractive method for multiplexed assays where several biomarkers are determined at once. The analysis of the 
three patient samples proved that the PeCAPrA method was able to detect different levels of endogenous ProGRP.

Based on these result it is expected that peptide capture by anti-protein antibodies successfully can be applied to other protein biomarkers. The huge variety of and easy access to commercially available antibodies allows for a wide application area. Peptide capture using monoclonal antibodies targeting linear protein epitopes has great potential in targeted proteomic approaches as it might facilitate the enrichment of signature peptides without the need for time-consuming and expensive development of anti-peptide antibodies, independently from batch-to-batch variations.

\section{Materials and methods}

\section{Protein standards and chemicals}

Cloned ProGRP isoform 1 and anti-ProGRP (monoclonal antibody E146, E149 and M18) were provided by the Central Laboratory, Norwegian Radium Hospital, Oslo University Hospital (Oslo, Norway). Trypsin (TPCK treated, from bovine pancreas, sequencing grade), Lys-C (from Lysobacter enzymogenes), and formic acid (FA) were purchased from Sigma Aldrich (St. Louis, MO, USA). All other chemicals used were of analytical grade.

\section{Serum samples}

Human serum from healthy subjects was obtained from Oslo University Hospital, Ullevål (Oslo, Norway), and serum samples from cancer patients were supplied by the Norwegian Radium Hospital, Oslo University Hospital. All serum samples were stored at $-30^{\circ} \mathrm{C}$. The use of patient samples for our research purposes was approved by the Norwegian Regional Committee for Medical Research Ethics (REK, http://helseforskning.etikkom.no). Informed consent was obtained from all subjects. Methods used to analyze all serum samples were in accordance with relevant guidelines and regulations.

\section{Solutions}

ProGRP isoform 1 (AA 1-125 + 8) was cloned from human cDNA (OriGene Technologies), expressed in Escherichia coli (Promega) using pGEX-6P-3 constructs (GE Healthcare) and purified as described elsewhere ${ }^{18}$. The concentration of the ProGRP stock solution was determined by absorbance at 280 $\mathrm{nm}$ (A280). Working solutions were prepared by dilution with $50 \mathrm{mM}$ ammonium bicarbonate buffer solution ( $A B C$ buffer) and stored at $4{ }^{\circ} \mathrm{C}$. 
Initially, complex samples were prepared by adding Lys-C or trypsin digested ProGRP to trypsin digested human serum from healthy subjects. The digested standards were added to the digested serum immediately before performing the extraction. In later experiments, spiked serum samples were prepared by adding intact ProGRP immediately before digestion with trypsin beads.

\section{Immobilization of trypsin to sepharose beads}

Coating of trypsin on sepharose beads was performed as described elsewhere ${ }^{30}$. In brief, NHSactivated sepharose beads (Sepharose ${ }^{\mathrm{TM}} 4$ Fast Flow, GE Healthcare, Uppsala, Sweden) were prewashed with 10 volumes of cold washing buffer ( $0.1 \mathrm{M}$ phosphate buffer, $\mathrm{pH} 7.8$ ). An equal volume of $20 \mathrm{mg} / \mathrm{mL}$ trypsin in coupling buffer $(0.1 \mathrm{M}$ ethanolamine, $0.25 \mathrm{M}$ benzamidine and $0.1 \mathrm{M}$ phosphate buffer, $\mathrm{pH} 7.8)$ was added and shaken $(800 \mathrm{rpm})$ at room temperature for $25 \mathrm{~min}$. Unbound trypsin was removed before modification buffer $(0.2 \mathrm{M}$ acetic acid $\mathrm{N}$ hydroxysuccinimide(NHS)esther in $0.1 \mathrm{M}$ phosphate buffer, $\mathrm{pH} 7.8$ ) was added (1:1 v/v) and shaken $(800 \mathrm{rpm})$ at room temperature for $20 \mathrm{~min}$. The excess NHS was deactivated by blocking buffer (0.1 $\mathrm{M}$ ethanolamine in $0.1 \mathrm{M}$ phosphate buffer, $\mathrm{pH}$ 8.0) $(1: 5 \mathrm{v} / \mathrm{v})$ and shaken $(800 \mathrm{rpm})$ at room temperature for $10 \mathrm{~min}$. The trypsin coated beads were stored in storage buffer (50 mM Tris pH 8.2, $1 \mathrm{mM}$ calcium chloride and $0.02 \%$ sodium azide) at $4^{\circ} \mathrm{C}$, protected from light. The final concentration of the immobilized trypsin beads are according to Freije et al ${ }^{30}$ about $16.3 \mathrm{mg} / \mathrm{mL}$.

\section{Enzymatic digestion}

In solution digestion of ProGRP isoform 1 diluted in freshly prepared $A B C$ buffer ( $50 \mathrm{mM}$ ) was initiated by adding freshly prepared protease (trypsin or Lys-C) to give an enzyme-to-protein ratio of 1:40 (w/w). On-beads digestion of spiked serum samples were initiated by adding freshly prepared trypsin to give an enzyme-to-antibody ratio of 1:5 (w/w). Samples were incubated over night at 800 rpm at $37^{\circ} \mathrm{C}$.

Digestion of protein precipitated serum samples were initiated by adding $30 \mu \mathrm{L}$ trypsin beads. Samples were incubated for 2 hours at $800 \mathrm{rpm}$ at $37^{\circ} \mathrm{C}$.

\section{Immobilization of monoclonal antibodies to magnetic beads}

Coating of monoclonal antibodies on magnetic beads was performed as described elsewhere ${ }^{31}$, using $1 \mathrm{mg}$ of antibody to $50 \mathrm{mg}$ of magnetic beads (Dynabeads M280 tosylactivated, Life Technology, Invitrogen Dynal, Oslo, Norway). To ensure the right orientation of the antibodies on the beads, the antibodies were added hydrocholorid acid $(\mathrm{HCl})$ to $\mathrm{pH} 2.5$ and incubated for 1 hour on ice, before coupling at pH 9.5 overnight at $37^{\circ} \mathrm{C}$. The coupling volume was $1 \mathrm{~g}$ beads to $40 \mathrm{~mL}$ solution. To 
remove any unbound antibody, the beads were washed twice with $1 \%$ bovine serum albumin (BSA) in $0.1 \mathrm{M}$ tris hydrochloride buffer $(\mathrm{pH} \mathrm{7.5)}$ for 2 hours, and once overnight, at room temperature. Antibody coated magnetic bead were stored in the same buffer $(\mathrm{pH} 7.5)$ at $4^{\circ} \mathrm{C}$.

\section{Peptide capture}

To remove any unbound anti-ProGRP the antibody-coated magnetic beads were prewashed as described elsewhere ${ }^{14}$ : Desired volume of beads was washed with $1 \mathrm{~mL}$ PBS containing $0.05 \%$ Tween 20 , and re-dissolved in PBS, yielding a solution with the initial bead concentration, ready for use.

The immunoaffinity extraction was performed as follows using magnetic beads: Protein LoBind Eppendorf tubes containing the sample (an in solution digest of the protein standard or a protein precipitated, diluted and digested serum sample) were added $20 \mu \mathrm{L}$ of prewashed antibody-coated magnetic beads. To capture the peptides the Eppendorf tubes were rotated and shaken for 1 hour on a HulaMixer (Invitrogen), to facilitate the epitope-antibody interaction. The Eppendorf tubes were then placed in the magnetic rack (DynaMag-2 from Invitrogen) to collect the beads and remove the solution. The beads were then washed with $500 \mu \mathrm{L}$ of PBS containing $0.05 \%$ Tween $20,500 \mu \mathrm{L}$ of PBS, $300 \mu \mathrm{L}$ of Tris- $\mathrm{HCl}(\mathrm{pH} 7.4)$, and $300 \mu \mathrm{L}$ of $50 \mathrm{mM} \mathrm{ABC}$ buffer, prior to elution.

\section{Elution of extracted peptides}

After immunocapture, the elution step was used for enrichment by adding $15 \mu \mathrm{L}$ of freshly prepared $2 \%$ formic acid (FA) to the washed beads. Samples were shaken at room temperature for $5 \mathrm{~min}$, placed in the magnetic rack and the supernatant containing eluted peptides was transferred to new Protein LoBind eppendorf tubes. Additional $15 \mu \mathrm{L}$ of $2 \%$ FA was added to the beads, shaken for 5 min and the two supernatants were collected in the same tube. The combined eluates were directly injected into the LC-MS system.

\section{Preparation of serum samples}

Fifty $\mu$ l human serum was protein precipitated using cold acetonitrile $\left(-32^{\circ} \mathrm{C}\right)$ in a ratio of $1: 0.7(\mathrm{v} / \mathrm{v})$ with subsequent vortexing for $1 \mathrm{~min}$ and centrifugation at $10000 \mathrm{rpm}$ for $10 \mathrm{~min}$. The supernatant was then transferred to new Protein LoBind Eppendorf tubes and diluted 1:40 with ABC buffer (50 $\mathrm{mM}$ ) to ensure optimal digestion conditions. Digestion was performed by adding $30 \mu \mathrm{L}$ trypsin beads solution and the samples were incubated for 2 hours at $37^{\circ} \mathrm{C}$. The samples were then centrifuged to sediment the beads and the solution was transferred to new Protein LoBind eppendorf tubes. Extraction and elution of bound peptides were then performed as described above. 


\section{Nano LC-MS/MS analysis}

Two different nanoLC-MS systems were used: an LTQ Discovery Orbitrap MS and a TSQ Quantiva, both from Thermo Fischer (Rockford, IL, US). The same columns and mobile phases were used for both systems. The samples were trapped on a C18 Acclaim PepMap 100 enrichment column (300 $\mu \mathrm{m}$ i.d. $x 5 \mathrm{~mm}, 5 \mu \mathrm{m}$; Thermo Fischer) and further separated on a C18 Acclaim PepMap 100 analytical column (75 $\mu$ m i.d. $\times 15 \mathrm{~cm}, 3 \mu \mathrm{m}$; Thermo Fischer). The loading buffer consisted of $20 \mathrm{mM}$ FA:MeCN $(97: 3, v / v)$, and the mobile phases consisted of A: $20 \mathrm{mM} \mathrm{FA:MeCN}(95: 5, \mathrm{v} / \mathrm{v})$ and B: $20 \mathrm{mM}$ FA:MeCN $(5: 95, v / v)$. An automatic filtration of the samples were also performed as described elsewhere using a steel filter (Replacement screen, $1 / 16$ " from Teknolab, Norway) ${ }^{32}$.

\section{LTQ-Orbitrap}

Twenty $\mu \mathrm{L}$ of each sample was injected into the Dionex 3000 ultimate chromatographic system. The loading mobile phase delivered the samples to the trap column with a flow rate of $10 \mu \mathrm{L} / \mathrm{min}$ for 4 min. The analytes were then backflushed to the analytical by the mobile phases with a flow rate of $0.300 \mu \mathrm{L} / \mathrm{min}$. Two different linear gradients were run; a short gradient from 0 to $50 \% \mathrm{~B}$ in $18 \mathrm{~min}$, then increased to $100 \% \mathrm{~B}$ for 2 min before switching back to $100 \mathrm{~A}$ in order to regenerate the column, and a longer gradient from 0 to $50 \%$ B in $60 \mathrm{~min}$, and then increased to $100 \%$ for $4 \mathrm{~min}$ before switching back to $100 \% \mathrm{~A}$ in order to regenerate the column. The total analysis time per run was either 31 or $89 \mathrm{~min}$. The nanospray ionization source was operated in the positive ionization mode and the spray voltage was set to $2.2 \mathrm{kV}$. The heated capillary was kept at $150{ }^{\circ} \mathrm{C}$. The capillary voltage was set at $45 \mathrm{~V}$, and the tube lens offset was $100 \mathrm{~V}$. Data-dependent acquisition was performed in the orbitrap mass analyser at a resolution of 30000 over a mass range between $\mathrm{m} / \mathrm{z}$ 300-2000 Da with charge state disabled. Up to six of the most intense ions per scan were fragmented by collision induced dissociation (CID) at $35 \%$ relative collision energy, activation time of $30 \mathrm{~ms}$, and analysed in the linear ion trap. The fragmented $\mathrm{m} / \mathrm{z}$ values were dynamically excluded for $15 \mathrm{~s}$ to minimize the extent of repeat sequencing of peptides and to fragment lower intensity $\mathrm{m} / \mathrm{z}$ values.

\section{TSQ Quantiva}

Twenty $\mu \mathrm{L}$ of each sample was injected into the Dionex 3000 ultimate chromatographic system. The loading mobile phase delivered the samples to the trap column with a flow rate of $10 \mu \mathrm{L} / \mathrm{min}$ for 5 min. The analytes were then backflushed from the trap column on to the analytical with a linear gradient (starting after $5 \mathrm{~min}$ ) from 0 to $50 \%$ mobile phase $B$ (flow rate $0.3 \mu \mathrm{L} / \mathrm{min}$ ) in $10 \mathrm{~min}$. The column was regenerated for 10 min with $100 \%$ mobile phase A before injecting the next sample. The column oven temperature was set to $35^{\circ} \mathrm{C}$. Selected reaction monitoring (SRM) was performed by a 
TSQ quantiva equipped with a nano-ESI source in positive mode. The spray voltage was $2250 \mathrm{~V}$ and the heated capillary was kept at $350{ }^{\circ} \mathrm{C}$. Nitrogen was used as sweep gas ( 2 arbitrary units). The epitope peptide was fragmented at $35 \mathrm{~V}$ in the collision cell (argon) and selected fragments were transferred to Q3 $(1005.45 \rightarrow 595.2,913.3,1028.3,1398.5)$.

\section{Data interpretation}

The MS raw files were processed with Proteome Discoverer 1.4 (Thermo Fischer), using the SEQUEST algorithm, searching against a ProGRP database generated from the sequence obtained from UniProt (January, 2015). Up to five missed cleavages were considered using trypsin, Lys-C and chymotrypsin as enzymes. Methionine oxidation was chosen as variable modification. The initial parent and fragment ion maximum mass deviation was set to $10 \mathrm{ppm}$ and $0.8 \mathrm{Da}$, respectively.

The Thermo Scientific Xcalibur software version 2.1 (Thermo Fischer) was used to manually extract ion chromatograms (XICs), peak area and signal intensities of selected tryptic peptides.

The extraction yield was calculated from the analysis of both the bound (eluate) and unbound (supernatant) fraction of the epitope peptide.

Evaluation of statistical significance was performed using a paired sample $t$-test, with a cut-off value of 0.05 .

\section{In silico digestion and similarity search}

The sequence for ProGRP isoform 1 was acquired through The National Center for Biotechnology Information (NCBI) database (accession number NP_002082.2). In silico digests (Protein Prospector version 5.18.1) were performed using the following parameters: trypsin, chymotrypsin or Lys-C digest, zero missed cleavages, no modifications, peptide mass 400-2000, and minimum peptide length of five amino acids. The results were used to determine which protease should be used to generate a zero missed cleavage peptide containing the intact epitope of mAb E146 and mAb E149/M18.

To investigate if the epitope containing zero missed cleavage peptides solely originated from ProGRP, an NCBI BLAST (National Center for Biotechnology Information, Basic Local Alignment Search Tool) sequence similarity search was performed and used to ensure specificity of the signature epitope peptides. BLASTP 2.6.0+ was used as an algorithm with protein sequences from NCBI's Reference Sequence Project (RefSeq) as the database of choice. In addition to Homo sapiens, the BLAST searches were performed on Mus musculus and Bos Taurus, because the antibodies and the proteases used were derived from these organisms respectively. 


\section{Data availability statement}

The datasets generated during and/or analysed during the current study are available from the corresponding author on reasonable request.

\section{REFERENCES}

1. Ackermann, B. L.; Berna, M. J., Expert review of proteomics 2007, 4, 175-186.

2. Matt, P.; Fu, Z.; Fu, Q.; Van Eyk, J. E., Physiological genomics 2008, 33, 12-17.

3. Fung, E. N.; Bryan, P.; Kozhich, A., Bioanalysis 2016, 8, 847-856.

4. Whiteaker, J. R.; Zhao, L.; Abbatiello, S. E.; Burgess, M.; Kuhn, E.; Lin, C.; Pope, M. E.; Razavi, M.; Anderson, N. L.; Pearson, T. W., Molecular \& Cellular Proteomics 2011, 10, M110. 005645.

5. Dittrich, J.; Becker, S.; Hecht, M.; Ceglarek, U., Proteomics-Clinical Applications 2015, 9, 5-16.

6. Boström, T.; Takanen, J. O.; Hober, S., Journal of Chromatography B 2016, 1021, 3-13.

7. Madian, A. G.; Rochelle, N. S.; Regnier, F. E., Analytical chemistry 2012, 85, 737-748.

8. Van Regenmortel, M. H. V., In Epitope Mapping Protocols: Second Edition, Schutkowski, M.; Reineke, U., Eds. Humana Press: Totowa, NJ, 2009; pp 3-20.

9. Schoenherr, R. M.; Zhao, L.; Ivey, R. G.; Voytovich, U. J.; Kennedy, J.; Yan, P.; Lin, C.; Whiteaker, J. R.; Paulovich, A. G., Proteomics 2016, 16, 2141-2145.

10. Halvorsen, T. G.; Reubsaet, L., TrAC Trends in Analytical Chemistry 2017, 95, 132-139.

11. Schoenherr, R. M.; Saul, R. G.; Whiteaker, J. R.; Yan, P.; Whiteley, G. R.; Paulovich, A. G., Molecular \& Cellular Proteomics 2015, 14, 382-398.

12. Zhao, C.; Trudeau, B.; Xie, H.; Prostko, J.; Fishpaugh, J.; Ramsay, C., Proteomics 2014, 14, 1311-1321.

13. Opuni, K. F.; Al-Majdoub, M.; Yefremova, Y.; El-Kased, R. F.; Koy, C.; Glocker, M. O., Mass spectrometry reviews 2018, 229-241.

14. Torsetnes, S. B.; Nordlund, M. S.; Paus, E.; Halvorsen, T. G.; Reubsaet, L. O., Journal of proteome research 2012, 12, 412-420.

15. Torsetnes, S. B.; Levernæs, M. S.; Broughton, M. N.; Paus, E.; Halvorsen, T. G.; Reubsaet, L. o., Analytical chemistry 2014, 86, 6983-6992.

16. Lange, V.; Picotti, P.; Domon, B.; Aebersold, R., Mol. Syst. Biol. 2008, 4, 222.

17. Nordlund, M. S.; Warren, D. J.; Laerdahl, J. K.; Paus, E., Tumor Biology 2009, 30, 265-275.

18. Nordlund, M. S.; Fermer, C.; Nilsson, O.; Warren, D. J.; Paus, E., Tumor Biology 2007, 28, 100110.

19. Nordlund, M. S.; Warren, D. J.; Nustad, K.; Bjerner, J.; Paus, E., Clinical chemistry 2008, 54, 919-922.

20. Razavi, M.; Frick, L. E.; LaMarr, W. A.; Pope, M. E.; Miller, C. A.; Anderson, N. L.; Pearson, T. W., Journal of proteome research 2012, 11, 5642-5649.

21. Anderson, N. L.; Razavi, M.; Pearson, T. W.; Kruppa, G.; Paape, R.; Suckau, D., Journal of proteome research 2012, 11, 1868-1878. 
22. Anderson, N. L.; Anderson, N. G.; Haines, L. R.; Hardie, D. B.; Olafson, R. W.; Pearson, T. W., Journal of proteome research 2004, 3, 235-244.

23. Whiteaker, J. R.; Zhao, L.; Anderson, L.; Paulovich, A. G., Molecular \& Cellular Proteomics 2010, 9, 184-196.

24. Xu, Q.; Zhu, M.; Yang, T.; Xu, F.; Liu, Y.; Chen, Y., Clinica Chimica Acta 2015, 448, 118-123.

25. Razavi, M.; Anderson, N. L.; Pope, M. E.; Yip, R.; Pearson, T. W., New biotechnology 2016, 33, 494-502.

26. Switzar, L.; Giera, M.; Niessen, W. M., Journal of proteome research 2013, 12, 1067-1077.

27. Rossetti, C.; Abdel Qader, A.; Halvorsen, T. G.; Sellergren, B. r.; Reubsaet, L. o., Analytical chemistry 2014, 86, 12291-12298.

28. Winther, B.; Moi, P.; Paus, E.; Reubsaet, J. L. E., Journal of separation science 2007, 30, 26382646.

29. Levernæs, M. C. S.; Broughton, M. N.; Reubsaet, L.; Halvorsen, T. G., Journal of Chromatography B 2017, 1055, 51-60.

30. Freije, J. R.; Mulder, P. P.; Werkman, W.; Rieux, L.; Niederlander, H. A.; Verpoorte, E.; Bischoff, R., Journal of proteome research 2005, 4, 1805-1813.

31. Paus, E.; Nustad, K., Clin. Chem. 1989, 35, 2034-2038.

32. Svendsen, K. O.; Larsen, H. R.; Pedersen, S. A.; Brenna, I.; Lundanes, E.; Wilson, S. R., Journal of separation science 2011, 34, 3020-3022.

\section{ACKNOWLEDGMENT}

This work has been performed within DIATECH@UiO, a strategic research initiative at the Faculty of Mathematics and Natural Sciences, University of Oslo, Oslo, Norway.

\section{AUTHOR INFORMATION}

\section{Corresponding author}

*Tel: +47 22855735. Fax: +47 22854402. E-mail: t.g.halvorsen@farmasi.uio.no

Postal address: Dept. Pharm. Chemistry, PO Box 1068 Blindern, 0316 Oslo, Norway

\section{Author contributions}

The manuscript was written through contributions of all authors. All authors (Maren C. S. Levernæs, Bassem Farhat, Inger Oulie, Sazan S. Abdullah, Elisabeth Paus, Léon Reubsaet, and Trine G. Halvorsen) have given approval to the final version of the manuscript.

\section{Competing interests}

The authors declare that they have no competing interests. 


\section{Supporting information}

\section{Exploring peptide capture by anti-protein antibodies for LC- MS-based biomarker determination}

Maren C. S. Levernæs ${ }^{1}$, Bassem Farhat ${ }^{1}$, Inger Oulie ${ }^{1}$, Sazan S. Abdullah ${ }^{1}$, Elisabeth Paus ${ }^{2}$, Léon Reubsaet $^{1}$, Trine G. Halvorsen ${ }^{1 *}$

${ }^{1}$ Department of Pharmaceutical Chemistry, School of Pharmacy, University of Oslo, Norway

${ }^{2}$ Department of Medical Biochemistry, Norwegian Radium Hospital, Oslo University Hospital, Norway 
A

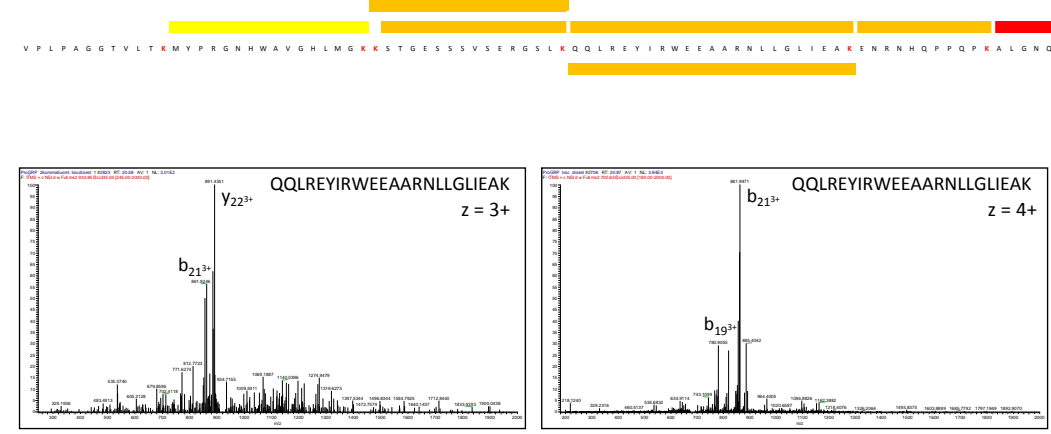

B
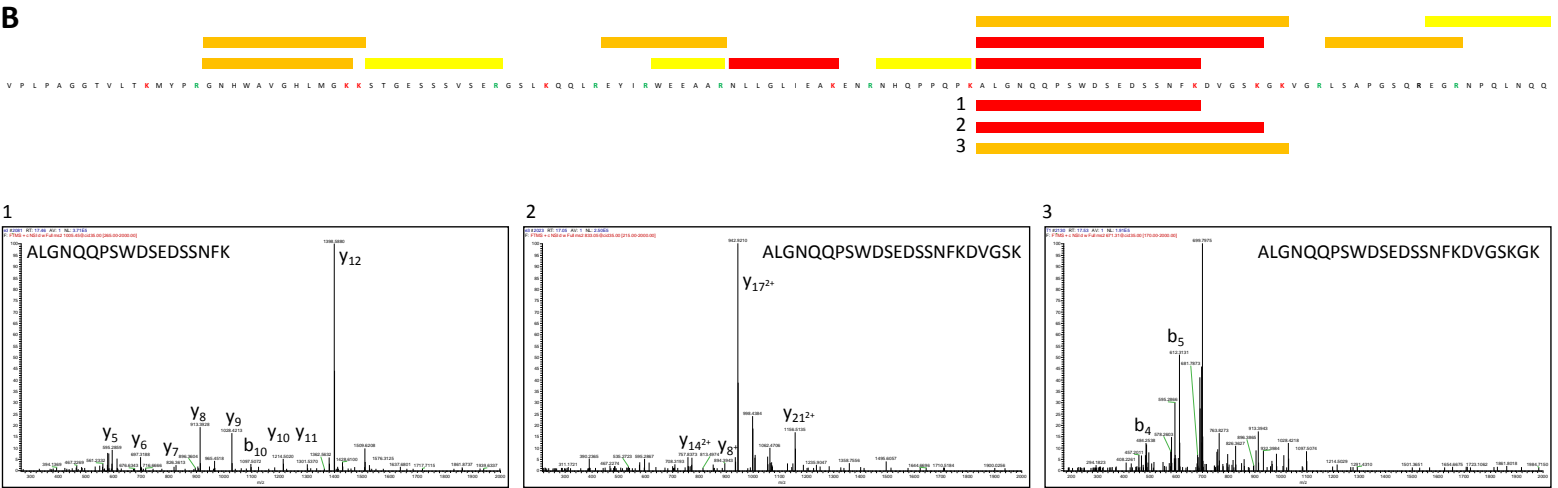

C
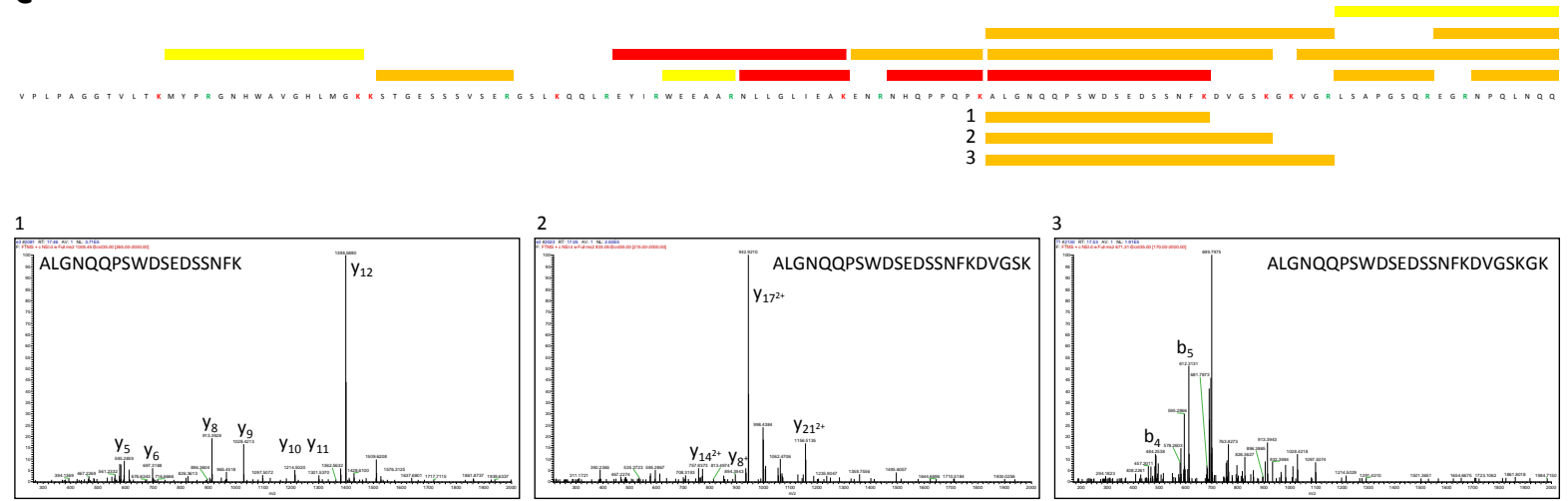

Figure S1. Proof of concept for E146 (A), M18 (B) and E149 (C). Peptides generated from an in solution digest of ProGRP (Lys-C digest in A and trypsin digests in B and C) are displayed above the sequence and peptides eluted from the antibody after PeCAPrA are displayed below the sequence. Lys- $C$ cleavages sites are marked with red (lysine) and trypsin cleavages sites are marked with red (lysine) and green (argenine) letters. Zero missed cleavages peptides are closest to the sequence. Signal intensity: - low, - medium and - high. The MS/MS spectra confirm the identity of the peptides identified in the eluate. 


\section{Optimization of protein precipitation}

There are several methods that are commonly used to reduce the complexity of a serum sample prior to digestion. The most used serum depletion methods in SISCAPA and related assays are depletion by filters ${ }^{22,33}$, columns ${ }^{24,34}$ and protein precipitation ${ }^{27,35}$. Protein precipitation has previously been used in the sample clean-up of ProGRP ${ }^{27-28}$. Protein precipitation is a procedure that involves two steps that may contribute to variation and loss of analyte; precipitation and evaporation. Winther et $\mathrm{al}^{28}$ and Rossetti et $\mathrm{al}^{27}$ have previously demonstrated that the protein precipitation step can be a challenge and cause of variation in the determination of ProGRP. In order to reduce the between sample variation originating from the protein precipitation step the following was investigated; 1) digestion directly in the diluted acetonitrile fraction without the evaporation step and 2) optimization of the amount of acetonitrile used for precipitation.

There is some documentation that indicates that trypsin is unaffected by the presence of acetonitrile $\mathrm{e}^{30,36}$, and dilution of the acetonitrile fraction (1:20 and 1:40) after protein precipitation was thus investigated as it would remove one source of analyte loss; the evaporation step. Dilution of the acetonitrile fraction with $A B C$ buffer in a ratio of 1:40 provided the highest signal intensity and the lowest RSD values (data not shown).

To ensure that the majority of ProGRP remained in the solution after protein precipitation, an optimization of the amount of acetonitrile used was performed (data not shown). The signal intensity of the epitope peptide reached its maximum when serum was precipitated with a ratio of 1:0.7. This ratio is slightly lower than used for ProGRP isoform 1 previously $(1: 0.75)^{37}$. The observed difference may be due to the following dilution of the acetonitrile fraction with $A B C$ buffer $(1: 40)$ prior to digestion in this work. These common efforts increased the signal intensity and reduced the between sample variation from $30-50 \%$ RSD to $10 \%$ RSD (data not shown). 


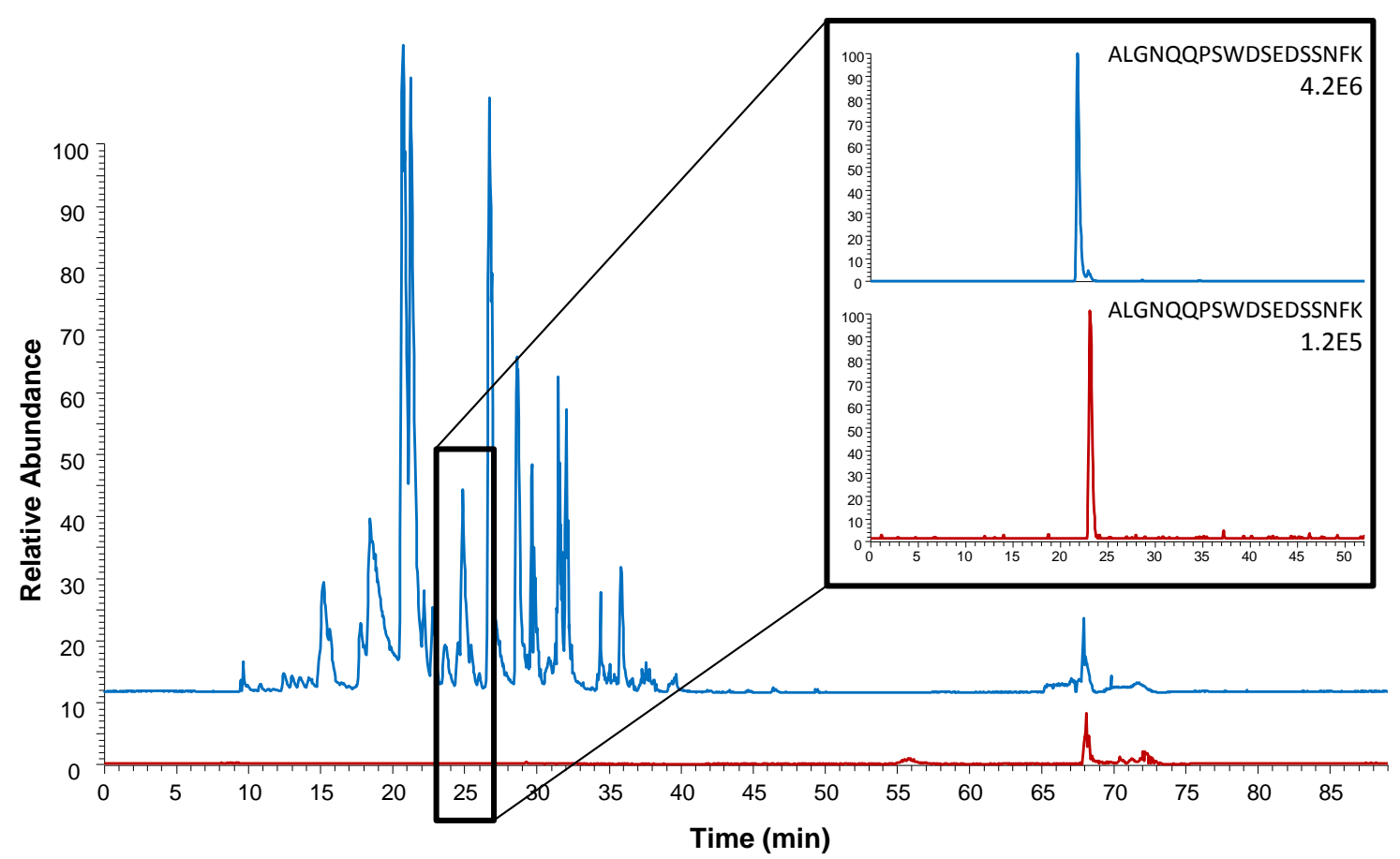

Figure S2. Comparison of basepeak chromatograms (full scan Orbitrap analysis) after intact protein extraction (blue) and epitope peptide extraction (PeCAPrA; red). Extracted ion chromatograms of the epitope signature peptide (ALGNQQPSWDSEDSSNFK, $\mathrm{m} / \mathrm{z}$ 1005.45) are shown on the right.
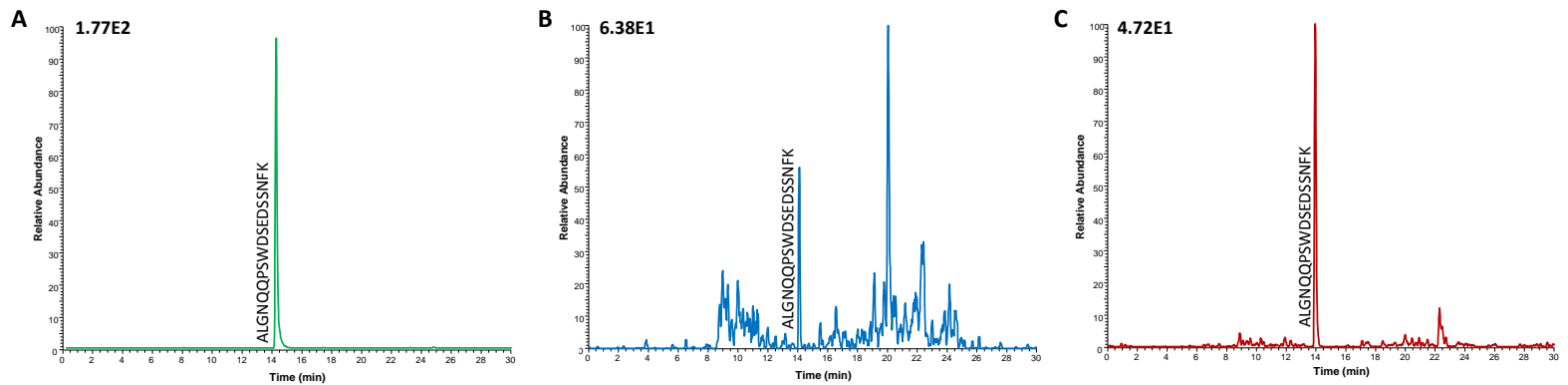

Figure S3. Extracted ion chromatograms of the epitope peptide after the analysis of serum samples from a spiked standard (A), a healthy individual (B) and from a patient with SCLC (C). 


\section{References}

1. Anderson, N. L.; Anderson, N. G.; Haines, L. R.; Hardie, D. B.; Olafson, R. W.; Pearson, T. W., J. Proteome Res. 2004, 3, 235-244.

2. Whiteaker, J. R.; Zhao, L.; Zhang, H. Y.; Feng, L.-C.; Piening, B. D.; Anderson, L.; Paulovich, A. G., Anal. Biochem. 2007, 362, 44-54.

3. Xu, Q.; Zhu, M.; Yang, T.; Xu, F.; Liu, Y.; Chen, Y., Clin. Chim. Acta 2015, 448, 118-123.

4. Ahn, Y. H.; Lee, J. Y.; Lee, J. Y.; Kim, Y.-S.; Ko, J. H.; Yoo, J. S., J. Proteome Res. 2009, 8, 42164224.

5. Kushnir, M. M.; Rockwood, A. L.; Roberts, W. L.; Abraham, D.; Hoofnagle, A. N.; Meikle, A. W., Clin. Chem. 2013, 59, 982-990.

6. Rossetti, C.; Abdel Qader, A.; Halvorsen, T. G.; Sellergren, B. r.; Reubsaet, L. o., Anal. Chem. 2014, 86, 12291-12298.

7. Winther, B.; Moi, P.; Paus, E.; Reubsaet, J. L. E., J. Sep. Sci. 2007, 30, 2638-2646.

8. Strader, M. B.; Tabb, D. L.; Hervey, W. J.; Pan, C.; Hurst, G. B., Anal. Chem. 2006, 78, 125-134.

9. $\quad$ Freije, J. R.; Mulder, P. P.; Werkman, W.; Rieux, L.; Niederlander, H. A.; Verpoorte, E.; Bischoff, R., J. Proteome Res. 2005, 4, 1805-1813.

10. Rossetti, C.; Świtnicka-Plak, M. A.; Halvorsen, T. G.; Cormack, P. A.; Sellergren, B.; Reubsaet, L., Sci. Rep. 2017, 7, 44298. 